\title{
Model reduction of semiaffinely parameterized partial differential equations by two-level affine approximation
}

\author{
Toni Lassila ${ }^{\mathrm{a}}$, Gianluigi Rozza ${ }^{\mathrm{b}}$ \\ ${ }^{a}$ Department of Mathematics and Systems Analysis, Aalto University, P.O. Box 11100, FI-00076 Aalto, Finland. \\ ${ }^{b}$ Modelling and Scientific Computing, Mathematics Institute of Computational Science and Engineering, École Polytechnique \\ Fédérale de Lausanne, Station 8, EPFL, CH-1015 Lausanne, Switzerland.
}

\begin{abstract}
We propose an improvement to the reduced basis method for parametric partial differential equations. An assumption of affine parameterization leads to an efficient offline-online decomposition when the problem is solved for many different parametric configurations. We consider an advection-diffusion problem, where the diffusive term is nonaffinely parameterized and treated with a two-level affine approximation given by the empirical interpolation method. The offline stage and a posteriori error estimation is performed using the coarse-level approximation, while the fine-level approximation is used to perform a correction iteration that reduces the actual error of the reduced basis approximation while keeping the same certified error bounds.
\end{abstract}

\section{Résumé}

Réduction de modèle pour des équations aux dérivées partielles paramétrisées semi-affinement par une approximation affine à deux niveaux. On propose une amélioration de la méthode des bases réduites pour des équations aux dérivées partielles paramétriques. On utilise l'hypothèse de paramétrisation affine pour obtenir un problème ayant des formes bilinéaires indépendantes des paramètres et des fonctions scalaires qui dépendent des paramètres. Ceci mène à une décomposition offline-online (qui est) plus efficace lorsque le problème est résolu pour différentes configurations des paramètres. Toutefois, dans le cas général, la condition de paramétrisation affine n'est pas satisfaite. On considère un problème d'advection-diffusion où la matrice des coefficients d'advection est paramétrisée de manière affine et où on traite le terme diffusif nonaffine avec une approximation affine à deux niveaux obtenue avec une méthode d'interpolation empirique. La partie offline est effectuée en utilisant une approximation affine grossière alors qu'une approximation affine plus fine est utilisée pour accomplir une itération de correction.

\section{Version française abrégée}

On considère une équation paramétrique d'advection-diffusion: soit $\boldsymbol{\mu}$ un vecteur de paramètres, trouver $u(\boldsymbol{\mu}) \in X(\Omega)$ tel que $(1)$ soit vérifié pour tout $v \in X(\Omega)$, où $\Omega \subset \mathbb{R}^{d}$ est un domaine de Lipschitz borné et $X(\Omega):=H_{\Gamma_{d}}^{1}(\Omega)$ est l'espace des fonctions $H^{1}$ qui s'annulent sur le bord correspondant aux conditions de Dirichlet $\Gamma_{d} \subset \partial \Omega$ avec la norme usuelle de Sobolev $\|\cdot\|_{H^{1}}$. Les formes bilinéaires paramétriques sont définies par (2) où le champ de vitesse $\boldsymbol{w}(\boldsymbol{x})$ est à divergence nulle et, pour simplifier, ne dépend pas des paramètres, et la forme linéaire paramétrique est définie par $\mathcal{F}(v ; \boldsymbol{\mu}) \triangleq \int_{\Omega} f(\boldsymbol{x}, \boldsymbol{\mu}) v(\boldsymbol{x}) d \Omega$. On s'intéresse au modèle de réduction de (1) en utilisant la méthode des bases réduites [1,2]. De travaux précédents sur ce sujet sont présentés dans [3, 4]. Dans l'Annexe, on présente brièvement les détails de l'approximation par les bases réduites pour l'équation d'advection-diffusion.

Email addresses: toni.lassila@tkk.fi (Toni Lassila), gianluigi.rozza@epfl.ch (Gianluigi Rozza) 
Par la suite, on fait les hypothèses suivantes: (i) Le vecteur des paramètres $\boldsymbol{\mu} \in \mathcal{D} \subset \mathbb{R}^{P}$ est de dimension finie et le domaine des valeurs admissibles des paramètres $\mathcal{D}$ est borné; (ii) La fonction matricielle $\nu(\boldsymbol{x}, \boldsymbol{\mu})$ est positive et uniformément définie positive pour tout $\boldsymbol{x} \in \Omega$ et $\boldsymbol{\mu} \in \mathcal{D}$; (iii) La fonction matricielle $\chi(\boldsymbol{x}, \boldsymbol{\mu})$ et le membre de droite $f(\boldsymbol{x} ; \boldsymbol{\mu})$ peuvent être décomposés de manière affine par (3) pour des entiers $Q_{b}, Q_{f}$, des fonctions scalaires lisses $\Theta_{b}^{q}, \Theta_{f}^{q}$, des fonctions matricielles $\chi^{q}$ et des fonctions scalaires $f^{q}$; (iv) Il existe deux niveaux d'approximations affines (fin et grossier) de la fonction matricielle non-affine $\nu(\boldsymbol{x}, \boldsymbol{\mu})$ par $(4)$ et (5) où $\nu_{\text {coarse }}$ et $\nu_{\text {fine }}$ sont toutes les deux symétriques et définies positives, et les termes restants satisfont (6) pour chaque $\boldsymbol{\mu} \in \mathcal{D}$ pour certaines tolérances $0<\delta_{\text {fine }}^{\text {tol }} \ll \delta_{\text {coarse }}^{\text {tol }}$ Ces approximations peuvent être obtenues en utilisant l'interpolation empirique (EIM) introduite dans [5].

Comme la partie advective de nos équations est paramétrisée de manière affine alors que la partie de diffusion ne l'est pas, on dit que l'équation est paramétrisée de manière semi-affine. En replacant le coefficient de la matrice de diffusion non-affine $\nu$ par son approximation fine $\nu_{\text {fine }}$ dans $(1)$, on obtient le problème affin: trouver $u(\boldsymbol{\mu}) \in X(\Omega)$ par (7) pour tout $v \in X(\Omega)$, où la forme bilinéaire est définie en replacant $\nu$ avec $\nu_{\text {fine }}$ dans (2). La méthode des bases réduites peut alors être appliquée pour réaliser un schéma efficace de réduction. de modèle. En choisissant $\delta_{\text {coarse }}^{\text {tol }}$ assez bas, la solution de ce problème affine est une approximation de la solution de (1), mais typiquement, on trouve que le coût de la partie offline croît considérablement lorsque le nombre de termes dans l'expansion $Q_{\text {fine }}$, croît. La solution de (7) est alors approximée par la séquence de problèmes suivants. Dans un premier temps, trouver $u^{0}(\boldsymbol{\mu}) \in X(\Omega)$ par $(\mathrm{P} 0)$ pour tout $v \in X(\Omega)$. Alors, pour chaque $k=1,2, \ldots$ trouver $u^{k}(\boldsymbol{\mu}) \in X(\Omega)$ par $(\mathrm{Pk})$ pour tout $v \in X(\Omega)$. Il faudrait montrer que la suite $\left\{u^{k}\right\}_{k=1}^{\infty}$ converge vers la solution de (7). Le schéma des bases réduites peut alors être écrit pour les équations $(\mathrm{P} 0)$ et $(\mathrm{Pk})$ afin d'obtenir une itération de point fixe dans l'espace réduit qui prend la solution des bases réduites grossière et utilise l'information issue de l'approximation plus fine pour améliorer la qualité de l'approximation.

\section{Introduction}

We consider a parametric advection-diffusion equation: for a given vector of parameters $\boldsymbol{\mu}$, find $u(\boldsymbol{\mu}) \in$ $X(\Omega)$ s.t.

$$
\mathcal{A}(u, v ; \boldsymbol{\mu})+\mathcal{B}(u, v ; \boldsymbol{\mu})=\mathcal{F}(v ; \boldsymbol{\mu})
$$

for all $v \in X(\Omega)$, where $\Omega \subset \mathbb{R}^{d}$ is a bounded Lipschitz domain and $X(\Omega):=H_{\Gamma_{d}}^{1}(\Omega)$ is the space of $H^{1}$ functions that vanish on the Dirichlet part of the boundary $\Gamma_{d} \subset \partial \Omega$ with the usual Sobolev norm $\|\cdot\|_{H^{1}}$. The parametric bilinear forms are defined as

$$
\mathcal{A}(u, v ; \boldsymbol{\mu}) \triangleq \varepsilon \int_{\Omega} \nu(\boldsymbol{x}, \boldsymbol{\mu}) \nabla u(\boldsymbol{x}) \cdot \nabla v(\boldsymbol{x}) d \Omega, \quad \mathcal{B}(u, v ; \boldsymbol{\mu}) \triangleq \int_{\Omega} v(\boldsymbol{x}) \boldsymbol{w}(\boldsymbol{x}) \cdot \chi(\boldsymbol{x}, \boldsymbol{\mu}) \nabla u(\boldsymbol{x}) d \Omega
$$

where the advective velocity field $\boldsymbol{w}(\boldsymbol{x})$ is divergence-free and for simplicity does not depend on the parameter, and the parametric linear form is defined as $\mathcal{F}(v ; \boldsymbol{\mu}) \triangleq \int_{\Omega} f(\boldsymbol{x}, \boldsymbol{\mu}) v(\boldsymbol{x}) d \Omega$. We are interested in parametric model reduction of (1) using the reduced basis method [1,2]. An important part of the reduced model is providing certified a posteriori error bounds between an underlying finite element approximation and its reduced basis approximation. Previous works on this topic include [3, 4]. In the Appendix we briefly present the details of reduced basis approximation for the advection-diffusion equation.

We make the following assumptions: (i) The parameter vector $\boldsymbol{\mu} \in \mathcal{D} \subset \mathbb{R}^{P}$ is finite-dimensional and the admissible parameter range $\mathcal{D}$ is bounded; (ii) The matrix function $\nu(\boldsymbol{x}, \boldsymbol{\mu}) \in C^{0}\left(\mathcal{D} ; L^{\infty}\left(\Omega ; \mathbb{R}^{d \times d}\right)\right)$ is symmetric and uniformly positive definite for all $\boldsymbol{x} \in \Omega$ and $\boldsymbol{\mu} \in \mathcal{D}$; (iii) The matrix function $\chi(\boldsymbol{x}, \boldsymbol{\mu}) \in$ $C^{0}\left(\mathcal{D} ; L^{\infty}\left(\Omega ; \mathbb{R}^{d \times d}\right)\right)$ and the right-hand side $f(\boldsymbol{x} ; \boldsymbol{\mu}) \in C^{0}\left(\mathcal{D} ; L^{2}(\Omega)\right)$ can be decomposed affinely into

$$
\chi(\boldsymbol{x}, \boldsymbol{\mu})=\sum_{q=1}^{Q_{b}} \Theta_{b}^{q}(\boldsymbol{\mu}) \chi^{q}(\boldsymbol{x}), \quad f(\boldsymbol{x}, \boldsymbol{\mu})=\sum_{q=1}^{Q_{f}} \Theta_{q}^{f}(\boldsymbol{\mu}) f^{q}(\boldsymbol{x})
$$

for some suitable integers $Q_{b}, Q_{f}$ and smooth, computable scalar functions $\Theta_{b}^{q}$, $\Theta_{f}^{q}$ and matrix functions $\chi^{q}$ and scalar functions $f^{q}$; (iv) There exist two levels of affine approximations (fine and coarse) of the nonaffine 
matrix function $\nu(\boldsymbol{x}, \boldsymbol{\mu})$ s.t.

$$
\nu(\boldsymbol{x}, \boldsymbol{\mu})=\sum_{q=1}^{Q_{\text {fine }}} \Theta_{\text {fine }}^{q}(\boldsymbol{\mu}) \nu_{\text {fine }}^{q}(\boldsymbol{x})+\varepsilon_{\text {fine }}(\boldsymbol{x}, \boldsymbol{\mu})=\nu_{\text {fine }}(\boldsymbol{x}, \boldsymbol{\mu})+\varepsilon_{\text {fine }}(\boldsymbol{x}, \boldsymbol{\mu})
$$

and

$$
\nu(\boldsymbol{x}, \boldsymbol{\mu})=\sum_{q=1}^{Q_{\text {coarse }}} \Theta_{\text {coarse }}^{q}(\boldsymbol{\mu}) \nu_{\text {coarse }}^{q}(\boldsymbol{x})+\varepsilon_{\text {coarse }}(\boldsymbol{x}, \boldsymbol{\mu})=\nu_{\text {coarse }}(\boldsymbol{x}, \boldsymbol{\mu})+\varepsilon_{\text {coarse }}(\boldsymbol{x}, \boldsymbol{\mu})
$$

where $\nu_{\text {coarse }}$ and $\nu_{\text {fine }}$ are both symmetric and uniformly positive definite, and the remainder terms satisfy

$$
\left\|\varepsilon_{\text {fine }}(\cdot, \boldsymbol{\mu})\right\|_{L^{\infty}} \leq \delta_{\text {fine }}^{\text {tol }}, \quad\left\|\varepsilon_{\text {coarse }}(\cdot, \boldsymbol{\mu})\right\|_{L^{\infty}}<\delta_{\text {coarse }}^{\text {tol }}
$$

for every $\boldsymbol{\mu} \in \mathcal{D}$ for some prescribed tolerances $0<\delta_{\text {fine }}^{\text {tol }} \ll \delta_{\text {coarse }}^{\text {tol }}$. These approximations can be obtained by using the empirical interpolation method (EIM) introduced in [5].

Because the advective part of our equations is affinely parameterized while the diffusive part is not, we say that the equation is semiaffinely parameterized. By replacing the nonaffine diffusive coefficient matrix $\nu$ with its fine level approximation $\nu_{\text {fine }}$ in (1) we obtain the affine problem to find $u(\boldsymbol{\mu}) \in X(\Omega)$ s.t.

$$
\mathcal{A}_{\text {fine }}(u, v ; \boldsymbol{\mu})+\mathcal{B}(u, v ; \boldsymbol{\mu})=\mathcal{F}(v ; \boldsymbol{\mu})
$$

for all $v \in X(\Omega)$, where the affinely parameterized bilinear form is defined by replacing $\nu$ with $\nu_{\text {fine }}$ in (2).

If the reduced basis method is applied directly to (7), the cost of computing the coercivity lower bound $\alpha_{\mathrm{LB}}$ is high since the workload scales polynomially in $Q_{\text {fine }}$, i.e. certifying the reduced solution is expensive. The solution of (7) is therefore approximated by the following sequence of problems. In the first step, find $u^{0}(\boldsymbol{\mu}) \in X(\Omega)$ s.t.

$$
\mathcal{A}_{\text {coarse }}\left(u^{0}, v ; \boldsymbol{\mu}\right)+\mathcal{B}\left(u^{0}, v ; \boldsymbol{\mu}\right)=\mathcal{F}(v ; \boldsymbol{\mu})
$$

for all $v \in X(\Omega)$. Then for each $k=1,2, \ldots$ find $u^{k}(\boldsymbol{\mu}) \in X(\Omega)$ s.t.

$$
\mathcal{A}_{\text {coarse }}\left(u^{k}, v ; \boldsymbol{\mu}\right)+\mathcal{B}\left(u^{k}, v ; \boldsymbol{\mu}\right)=\mathcal{F}(v ; \boldsymbol{\mu})+\mathcal{A}_{\text {coarse }}\left(u^{k-1}, v ; \boldsymbol{\mu}\right)-\mathcal{A}_{\text {fine }}\left(u^{k-1}, v ; \boldsymbol{\mu}\right)
$$

for all $v \in X(\Omega)$. We shall show that the sequence $\left\{u^{k}\right\}_{k=1}^{\infty}$ converges to the solution of (7) when $\delta_{\text {coarse }}^{\text {tol }}$ is sufficiently small. The cost of the offline stage for the problems (P0) and (Pk) is much smaller since $Q_{\text {coarse }} \ll Q_{\text {fine }}$. Once the coarse-level reduced system with certified error bounds has been obtained, from the convergence of the fixed point algorithm we also get a certificate for the fine-level problem with the same error bounds. Thus the reduced basis method has been split into two levels of approximation detail: the coarse-level tolerance $\delta_{\text {coarse }}^{\text {tol }}$ controls the certification of the error bounds and cost of the offline stage, while the $\delta_{\text {fine }}^{\text {tol }}$ controls the actual error of the reduced basis solution. The user of the reduced model can then balance between the computational time spent in preparing the reduced model and the size of the certified error bounds without sacrificing the accuracy of the reduced model.

\section{Convergence proof for the correction iteration}

Theorem 2.1. Assume that the coarse level approximation $\nu_{\text {coarse }}$ has tolerance at most $\delta_{\text {coarse }}^{\text {tol }}<\frac{\alpha_{L B}}{2 \varepsilon d}$, where $\alpha_{L B}>0$ is the uniform lower bound for the coercivity constant of the coarse level problem, i.e.

$$
0<\alpha_{L B} \leq \alpha(\boldsymbol{\mu})=\inf _{v \in X(\Omega)} \frac{\mathcal{A}_{\text {coarse }}(v, v ; \boldsymbol{\mu})+\mathcal{B}(v, v ; \boldsymbol{\mu})}{\|v\|_{H^{1}}^{2}} \quad \text { for all } v \in X(\Omega), \boldsymbol{\mu} \in \mathcal{D} .
$$

Then the sequence $\left\{u^{k}\right\}_{k=0}^{\infty}$ given by (P0) and (Pk) converges to a solution of (7). 
Proof. By subtracting $(\mathrm{P}(\mathrm{k}+1))$ and $(\mathrm{Pk})$ from each other implies that the difference $w:=u^{k+1}-u^{k}$ satisfies $\mathcal{A}_{\text {coarse }}(w, v)+\mathcal{B}(w, v)=\mathcal{G}^{k}(v)$, where $\mathcal{G}^{k}(v):=\varepsilon \int_{\Omega}\left(\nu_{\text {coarse }}-\nu_{\text {fine }}\right) \nabla\left(u^{k}-u^{k-1}\right) \cdot \nabla v d \Omega$. The standard stability result due to the Lax-Milgram theorem gives $\left\|u^{k+1}-u^{k}\right\|_{H^{1}} \leq \alpha^{-1}(\boldsymbol{\mu})\left\|\mathcal{G}^{k}\right\|_{H^{-1}}$, where $\alpha(\boldsymbol{\mu})>0$ is the parameter dependent coercivity constant (8). We estimate

$$
\sup _{v \in X(\Omega)} \frac{\mathcal{G}^{k}(v)}{\|v\|_{H^{1}}} \leq \varepsilon\left\|\left(\nu_{\text {coarse }}-\nu_{\text {fine }}\right) \nabla\left(u^{k}-u^{k-1}\right)\right\|_{L^{2}} \leq 2 \varepsilon d \delta_{\text {coarse }}^{\text {tol }} \cdot\left\|u^{k}-u^{k-1}\right\|_{H^{1}},
$$

where in the last step we have used the fact that

$$
\left\|\nu_{\text {coarse }}(\cdot, \boldsymbol{\mu})-\nu_{\text {fine }}(\cdot, \boldsymbol{\mu})\right\|_{L^{\infty}} \leq \delta_{\text {coarse }}^{\text {tol }}+\delta_{\text {fine }}^{\text {tol }} \leq 2 \delta_{\text {coarse }}^{\text {tol }}, \quad \text { for all } \boldsymbol{\mu} \in \mathcal{D}
$$

according to assumptions (4), (5), and (6),

$$
\left\|u^{k+1}-u^{k}\right\|_{H^{1}} \leq \frac{2 \varepsilon d \delta_{\text {coarse }}^{\text {tol }}}{\alpha(\boldsymbol{\mu})}\left\|u^{k}-u^{k-1}\right\|_{H^{1}} \leq \frac{2 \varepsilon d \delta_{\text {coarse }}^{\text {tol }}}{\alpha_{\mathrm{LB}}}\left\|u^{k}-u^{k-1}\right\|_{H^{1}} .
$$

Thus the sequence converges in $X(\Omega)$ to the solution of $(1)$ when $\delta_{\text {coarse }}^{\text {tol }}<\frac{\alpha_{\mathrm{LB}}}{2 \varepsilon d}$ by contractivity.

\section{Numerical implementation}

For computational purposes the exact solutions of affine problems (P0) and (Pk) are approximated with the finite element method. Given a subspace $X_{h}(\Omega) \subset X(\Omega)$ of piecewise polynomial continuous functions defined on a discrete, regular mesh, we seek a sequence of discrete approximations $u_{h}^{k} \in X_{h}(\Omega), k=1,2, \ldots$, s.t.

$$
\mathcal{A}_{\text {coarse }}\left(u_{h}^{k}, v_{h} ; \boldsymbol{\mu}\right)+\mathcal{B}\left(u_{h}^{k}, v_{h} ; \boldsymbol{\mu}\right)=\mathcal{F}\left(v_{h} ; \boldsymbol{\mu}\right)+\mathcal{A}_{\text {coarse }}\left(u_{h}^{k-1}, v ; \boldsymbol{\mu}\right)-\mathcal{A}_{\text {fine }}\left(u_{h}^{k-1}, v ; \boldsymbol{\mu}\right)
$$

for all $v_{h} \in X_{h}(\Omega)$. We assume the dimension of the FE space, $\operatorname{dim}\left(X_{h}\right)=\mathcal{N}$, is chosen large enough so that the FE solution is an accurate representation of the true solution. The reduced basis method detailed in the Appendix can then be applied to obtain a reduced linear system of size $N \times N$

$$
\left(\sum_{q=1}^{Q_{\text {coarse }}} \Theta_{\text {coarse }}^{q}(\boldsymbol{\mu}) \underline{A}_{N}^{q}+\sum_{q=1}^{Q_{b}} \Theta_{b}^{q}(\boldsymbol{\mu}) \underline{B}_{N}^{q}\right) u_{N}^{k}=\left(\sum_{q=1}^{Q_{f}} \Theta_{f}^{q}(\boldsymbol{\mu}) F^{q}\right)+\left(\sum_{q=1}^{Q_{\text {coarse }}} \Theta_{\text {coarse }}^{q}(\boldsymbol{\mu}) \underline{A}_{N}^{q}-\sum_{q=1}^{Q_{\text {fine }}} \Theta_{\text {fine }}^{q}(\boldsymbol{\mu}) \underline{A}_{N}^{q}\right) u_{N}^{k-1}
$$

i.e. the reduced basis version of equation $(\mathrm{Pk})$. We have assumed here that the coarse and fine level approximations are hierarchical, that is, $\nu_{f}^{q}(\boldsymbol{x}) \equiv \nu_{c}^{q}(\boldsymbol{x})$ for all $q \leq Q_{c}$. The reduced basis system matrices $\underline{A}_{N}^{q}$ ( $Q_{\text {fine }}$ in total $), \underline{B}_{N}^{q}\left(Q_{b}\right.$ in total), and the vectors $F^{q}\left(Q_{f}\right.$ in total $)$ need to be assembled and stored in the offline stage. This is the only part of the offline stage that has complexity depending on $Q_{\text {fine. The }}$ rest of the offline stage depends only on $Q_{\text {coarse }}$. In the online stage, starting from an initial guess such as $u_{N}^{0}=[1,0,0, \ldots]^{t}$ we evaluate the coefficient functions depending only on the parameter, use the stored system matrices and right-hand sides to assemble the system (13), and solve to obtain the next iterate $u_{N}^{1}$. This fixed-point iteration is continued until a sufficient tolerance is achieved, $\left|u_{N}^{K}-u_{N}^{K-1}\right|<$ TOL. We can then reconstruct an approximation to the finite element solution of $(7)$ as $u_{h} \approx \sum_{n=1}^{N}\left[u_{N}^{K}\right]_{n} \Phi_{n}(\boldsymbol{x})$, where $\Phi_{n}$ are the orthonormalized reduced basis functions.

\section{Test case: airfoil in thermal flow}

Our test case involves the design of an airfoil in an exterior thermal flow that was considered in [3]:

$$
\begin{array}{r}
\int_{\Omega_{o}(\boldsymbol{\mu})}(\varepsilon \nabla u \cdot \nabla v+v \boldsymbol{w}(\boldsymbol{x}) \cdot \nabla u) d \Omega_{o}=\int_{\Omega_{o}(\boldsymbol{\mu})} f v d \Omega_{o} \quad \forall v \in H^{1}\left(\Omega_{o}(\boldsymbol{\mu})\right) \\
\quad \text { with } u=T_{0} \text { on } \Gamma_{\text {in }} \cup \Gamma_{\text {free }}, u=T_{1} \text { on } \Gamma_{\text {surf }}, \quad u=T_{2} \text { on the airfoil. }
\end{array}
$$




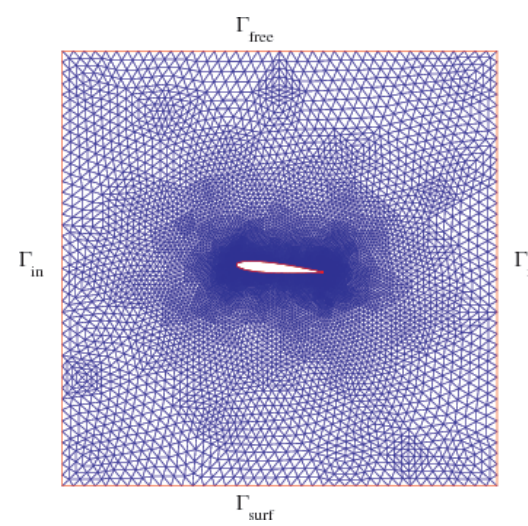

(a) Computational geometry
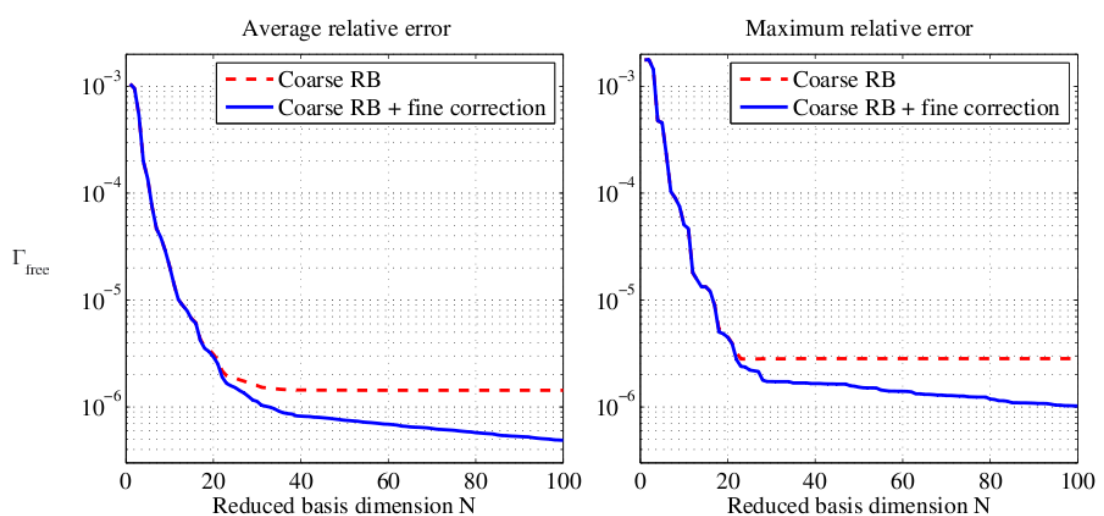

(b) Error $\left\|u_{h}-u_{h}^{N}\right\| /\left\|u_{h}\right\|$ of RB approximation over a random sample of $\boldsymbol{\mu} \in \mathcal{D}$

Figure 1: Finite element mesh on the computational reference domain and the reduced basis approximation errors

The reference geometry $\Omega$ is shown in Fig. 1(a). We parametrized the geometry around the airfoil $\Omega_{O}(\boldsymbol{\mu})=$ $T(\Omega, \boldsymbol{\mu})$ by free-form deformations (FFD) [6]: a $6 \times 6$ lattice of control points was placed around the airfoil and the closest four control points are allowed to move in the $x_{2}$-direction. This results in a polynomial geometric map with $P=4$ parameters $\boldsymbol{\mu}=\left\{\mu_{p}, p=1, . ., P\right\}$, representing the vertical displacements by the parameters range $\mathcal{D}=[-0.2,0.2]^{4}$. For the FEM computations $\mathcal{N}=15,718$ degrees of freedom were used.

The advective coefficient matrix is affinely parameterized as per assumptions. We chose $\varepsilon^{-1}=100=\mathrm{Pe}$ (convection dominated flow). A uniform lower bound for the coercivity constant $\alpha(\boldsymbol{\mu})>0.1$ then holds over the parameter range and the criterion for the coarse level tolerance is $\delta_{\text {coarse }}^{\text {tol }}<2.5 \mathrm{e}-4$. This tolerance was achieved by EIM in the example when $Q_{c}=76$. For the fine level expansion we used $\delta_{\text {fine }}^{\text {tol }}=1 \mathrm{e}-7$, which gives $Q_{f}=644$ terms. The advective part was affinely decomposed with $Q_{b}=11$ terms.

The cost of the offline stage is dominated by the construction of the lower bounds for the coercivity constant $\alpha(\boldsymbol{\mu})$. This involves the solution of $2\left(Q_{b}+Q_{c}\right)$ initial eigenproblems of size $\mathcal{N} \times \mathcal{N}$, and $K_{\max }$ steps of the algorithm each involves a linear programming problems of size $O\left(Q_{b}+Q_{c}\right)$. In addition we have an operation count of $O\left(\mathcal{N} K_{\max }\left(Q_{b}+Q_{c}\right)\right)$, see [7]. Thus at the coarse level the SCM has to perform $87 \%$ fewer initial eigensolves, and the cost of each iteration is similarly reduced. For this problem the SCM terminated after $K_{\max }=3$ steps. The coarse-level error bounds together with a training set of size $\left|\Xi_{\text {train }}\right|=1000$ were used to select a reduced basis with a total of $N_{\max }=100$ basis functions. The certificate obtained for the coarse-level problem was $\Delta_{N}(\boldsymbol{\mu}) \leq 1 \mathrm{e}-5$ for all $\boldsymbol{\mu} \in \mathcal{D}$.

In the online stage the reduced problem was solved with the coarse-level RB as an initial guess. Then the preassembled fine-level structures were used to drive the fixed-point correction iteration. Typically $4-5$ fixedpoint iterations were needed for convergence to TOL $=1 \mathrm{e}-9$. The error of the reduced basis approximation when compared to the full FEM solution is displayed in Fig. 1(b). We can observe a plateau effect [8], i.e. the convergence of the approximation stagnates early due to replacing the nonaffine diffusive tensor with its coarse approximation. The fine-level correction reduced the plateau effect while maintaining the certified error bounds at the coarse level.

\section{Conclusions}

The cost of certifying the solutions of the reduced basis method for nonaffinely parameterized problems can be considerably reduced by adopting a two-level strategy. We demonstrated the proposed method on a geometrically parameterized advection-diffusion equation. The savings in the offline stage were $87 \%$, mainly in terms of the number of eigensolutions needed for the successive constraint method. The fine-level correction improved the error of the final reduced basis solution and reduced the plateau effect typically observed in nonaffinely parameterized problems when the empirical interpolation tolerance is not sufficiently small. The certified error bounds were recovered from the coarse-level problem. 


\section{Acknowledgements}

We acknowledge the support of Prof. A. Quarteroni and Mr. A. Manzoni (EPFL) for some of the numerical tests, as well as Prof. A.T. Patera and Dr. D.B.P. Huynh (MIT) for the parts relating to empirical interpolation and the successive constraint method. G. Rozza's visit to Aalto University was part of the international visitor program 700304 funded by the Finnish Cultural Foundation and hosted by Prof. T. Eirola.

\section{Appendix: Reduced basis method for parametric PDEs}

The standard Galerkin finite element (FE) approximation of (1) is to find $u_{h} \in X_{h}(\Omega)$ s.t. $\mathcal{A}\left(u_{h}, v ; \boldsymbol{\mu}\right)+$ $\mathcal{B}\left(u_{h}, v ; \boldsymbol{\mu}\right)=\mathcal{F}(v ; \boldsymbol{\mu})$ for all $v \in X_{h}(\Omega)$, where $X_{h}$ is a FE space constructed by using e.g. piecewise linear shape functions on a discrete mesh. We assume $\operatorname{dim}\left(X_{h}(\Omega)\right)=\mathcal{N}$ is large enough so that the repeated assembly and solution of the FE system is prohibitively expensive for a multi-query context. In order to find an approximation to $u_{h}$ in an efficient and reliable way, we use Galerkin projection on a reduced subspace of basis functions. Let $\boldsymbol{\mu}^{1}, \ldots, \boldsymbol{\mu}^{N}$ be a collection of parameter vectors and define the reduced basis approximation space as $X_{h}^{N}:=\operatorname{span}\left\{u_{h}\left(\boldsymbol{\mu}^{n}\right): n=1, \ldots, N\right\}$, where each $u_{h}\left(\boldsymbol{\mu}^{n}\right) \in X_{h}$ is a FE solution for a given parameter value $\boldsymbol{\mu}^{n}$. The reduced basis formulation reads as follows: find $u_{h}^{N} \in X_{h}^{N}$ s.t. $\mathcal{A}\left(u_{h}^{N}, v ; \boldsymbol{\mu}\right)+\mathcal{B}\left(u_{h}^{N}, v ; \boldsymbol{\mu}\right)=\mathcal{F}(v)$, for all $v \in X_{h}^{N}$. If the parametric bilinear forms are affinely parametrized [2], that is to say of the form $\mathcal{A}(u, v ; \boldsymbol{\mu})=\sum_{q=1}^{Q_{a}} \Theta_{a}^{q}(\boldsymbol{\mu}) \mathcal{A}^{q}(u, v)$ and $\mathcal{B}(u, v ; \boldsymbol{\mu})=\sum_{q=1}^{Q_{b}} \Theta_{b}^{q}(\boldsymbol{\mu}) \mathcal{B}^{q}(u, v)$, the solution of the reduced basis problem splits into two stages. In the so-called offline stage we assemble and store once and for all the parameter-independent system matrices $\underline{A}^{q}$ and $\underline{B}^{q}$ of components $\left[\underline{A}^{q}\right]_{m, n}=$ $\mathcal{A}^{q}\left(\Phi_{n}, \Phi_{m}\right)$ and $\left[\underline{B}^{q}\right]_{m, n}=\mathcal{B}^{q}\left(\Phi_{m}, \Phi_{n}\right)$ using the global reduced basis functions $\Phi_{k}$, and similarly for the right-hand-sides. Then in the online stage for a given parameter $\boldsymbol{\mu}$ the parametric coefficients $\Theta_{a}^{q}(\boldsymbol{\mu}), \Theta_{b}^{q}(\boldsymbol{\mu})$ are evaluated and the reduced basis matrices $\underline{A}_{N}=\sum_{q=1}^{Q_{a}} \Theta_{a}^{q}(\boldsymbol{\mu}) \underline{A}^{q}, \quad \underline{B}_{N}=\sum_{q=1}^{Q_{b}} \Theta_{b}^{q}(\boldsymbol{\mu}) \underline{B}^{q}$ are assembled, and similarly for the right-hand-side. This linear system of dimension $N \times N$ is dense, but inexpensive to solve: the online complexity is independent of the FE solution dimension $\mathcal{N}$ [1].

To have confidence in our reduced model we require an efficient and computable a posteriori error estimator $\Delta_{N}(\boldsymbol{\mu})$ that measures the error: $\left\|u_{h}(\boldsymbol{\mu})-u_{h}^{n}(\boldsymbol{\mu})\right\| \leq \Delta_{n}(\boldsymbol{\mu})$ for all $\boldsymbol{\mu} \in \mathcal{D}$. In this case we say that the reduced model is certified. The standard estimator is based on the residual of the reduced basis solution $\Delta_{N}(\boldsymbol{\mu}):=\left\|\mathcal{F}(\cdot ; \boldsymbol{\mu})-\mathcal{A}\left(u_{h}^{N}, \cdot ; \boldsymbol{\mu}\right)-\mathcal{B}\left(u_{h}^{N}, \cdot ; \boldsymbol{\mu}\right)\right\|_{X_{h}^{\prime}(\Omega)} / \alpha_{\mathrm{LB}}(\boldsymbol{\mu})$, where $\alpha_{\mathrm{LB}}(\boldsymbol{\mu})$ is an online computable lower bound estimate for the parametric coercivity constant of the problem. To construct $\alpha_{\mathrm{LB}}(\boldsymbol{\mu})$ the successive constraint method (SCM) [7] can be used. For the details of implementing $\Delta_{N}(\boldsymbol{\mu})$ we refer to $[2,9]$.

\section{References}

[1] A. Patera, G. Rozza, Reduced Basis Approximation and A Posteriori Error Estimation for Parametrized Partial Differential Equations, Version 1.0, Copyright MIT 2006, to appear in (tentative rubric) MIT Pappalardo Graduate Monographs in Mechanical Engineering., 2010, available at http://augustine.mit.edu.

[2] G. Rozza, D. Huynh, A. Patera, Reduced basis approximation and a posteriori error estimation for affinely parametrized elliptic coercive partial differential equations, Arch. Comput. Methods Engrg. 15 (2008) 229-275.

[3] G. Rozza, T. Lassila, A. Manzoni, Reduced basis approximation for shape optimization in thermal flows with a parametrized polynomial geometric map, In: Einar Rønquist, Jan S. Hesthaven (Eds.) Proceedings of International Conference on Spectral and High-Order Methods, June 22-26, 2009, Trondheim, Norway. Lecture notes in Comp. Sci. Eng., Springer, 2010.

[4] T. Tonn, K. Urban, A reduced-basis method for solving parameter-dependent convection-diffusion problems around rigid bodies, In: P. Wesseling, E. Oñate, J. Périaux (Eds.), Proc. ECCOMAS CFD, Egmond aan Zee, The Netherlands, September 5-8, 2006.

[5] M. Barrault, Y. Maday, N. Nguyen, A. Patera, An 'empirical interpolation' method: application to efficient reduced-basis discretization of partial differential equations, C. R. Math. Acad. Sci. Paris 339 (9) (2004) 667-672.

[6] T. Sederberg, S. Parry, Free-form deformation of solid geometric models, Comput. Graph. 20(4) (1986) 151-160.

[7] D. Huynh, G. Rozza, S. Sen, A. Patera, A successive constraint linear optimization method for lower bounds of parametric coercivity and inf-sup stability costants, C. R. Acad. Sci. Paris. Sér. I Math. 345 (2007) 473-478.

[8] M. Grepl, Y. Maday, N. Nguyen, A. Patera, Efficient reduced-basis treatment of nonaffine and nonlinear partial differential equations, ESAIM Math. Modelling Numer. Anal. 41(3) (2007) 575-605.

[9] N. Nguyen, A posteriori error estimation and basis adaptivity for reduced-basis approximation of nonaffine-parametrized linear elliptic partial differential equations, J. Comp. Phys. 227 (2007) 983-1006. 